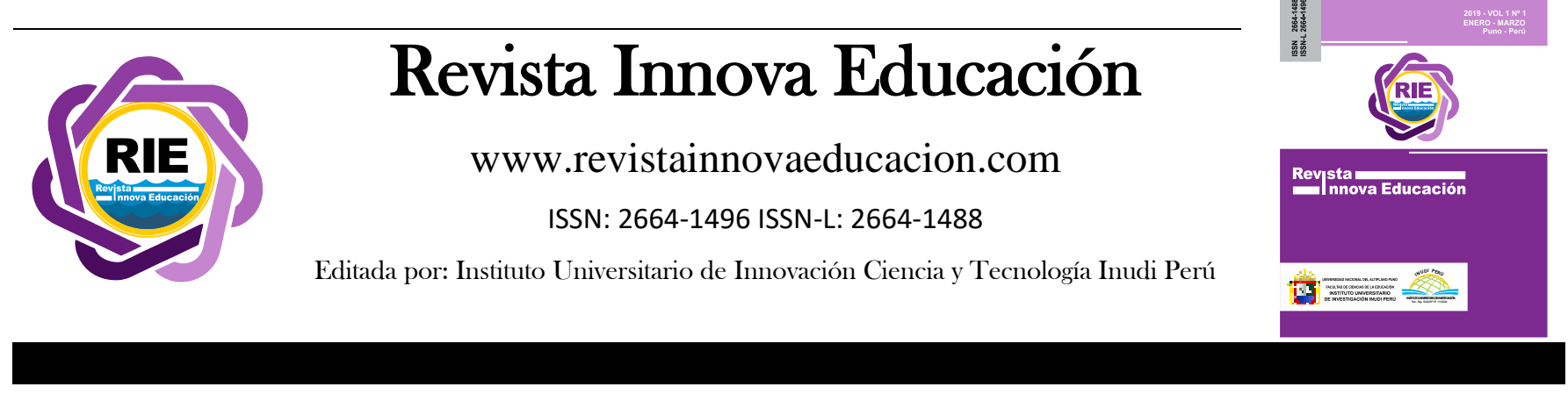

\title{
Compromiso organizacional y desempeño docente en las Instituciones de Educación Básica
}

\section{Organizational commitment and teaching performance in Basic Education Institutions}

Edwin Estrada 1

Universidad Nacional Amazónica de Madre de Dios, Puerto Maldonado - Madre de Dios, Perú https://orcid.org/0000-0003-4159-934X

\section{Helen Mamani}

Universidad Nacional Amazónica de Madre de Dios, Puerto Maldonado - Madre de Dios, Perú https://orcid.org/0000-0003-1735-8570

DOI: https://doi.org/10.35622/j.rie.2020.01.008

Recibido el 18/12/2019/ Aceptado el 25/01/2020 Publicado el 31/01/2020

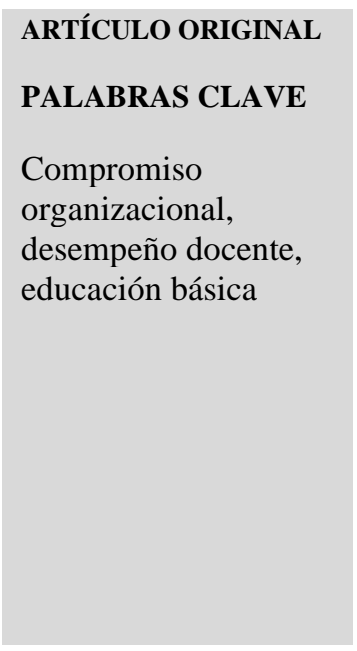

\section{KEYWORDS}

Organizational commitment, teaching performance, basic education
El objetivo fue establecer la relación que existe entre el compromiso organizacional y el desempeño docente en las Instituciones Educativas Urbanas del distrito de Las Piedras - 2018. El tipo de investigación fue no experimental, el diseño descriptivo correlacional de corte transversal. La población de estudio fue conformada por 106 docentes y la muestra por 83 docentes, cantidad que se obtuvo mediante muestreo probabilístico estratificado. Para recolectar información se utilizó el Cuestionario de Compromiso Organizacional de Meyer y Allen adaptado en el Perú por Oscar Martín Rivera Carrascal y el Cuestionario de Desempeño Docente. Los datos fueron consolidados en una base de datos para ser procesados mediante paquete estadístico Statical Package for the Social Sciences (SPSS). Los resultados indican que existe una relación fuerte, directa y significativa entre las variables compromiso organizacional y el desempeño docente. El coeficiente de correlación rho de Spearman es de 0,724 con un $\mathrm{p}$-valor inferior al nivel de significancia $(\mathrm{p}=0,000<0,05)$. Se concluye que mientras los docentes presenten un mayor compromiso organizacional, se desempeñarán de mejor manera y viceversa.

When economic growth is considered as a measure of the welfare of the population, it constitutes one of the basic objectives of economic policy; Therefore, the study of the factors or causes that originate it has been, and continues to be, one of the relevant topics in the economic discussion. The foregoing gave rise to the interest of determining whether public spending for education generated a positive or negative externality in the economic growth of the countries of the Andean region in the period 2000-2015. The study was conducted under the quantitative approach, specifically through the development of an econometric model. The main results show that in the

\footnotetext{
${ }^{1}$ Correspondencia: edwin5721@outlook.com
} 
study period, the countries of the Andean region developed under similar contexts and policies, where the characteristics and decisions of each country regarding public spending, although they differentiated them, in turn allowed to identify a joint reaction at the level of the region, regarding the results of social policies, concluding that public spending for education came to generate positive externalities in the economic growth of the Andean region.

\section{INTRODUCCIÓN}

En la actualidad se evidencia que existe una gran responsabilidad por parte del equipo directivo de las instituciones de educación básica de gestionar los recursos humanos que lideran, elaborar estrategias que posibiliten y favorezcan la articulación y trabajo colegiado de los docentes con la finalidad de concretizar los objetivos institucionales trazados en los documentos de gestión, tanto de corto y largo plazo.

Existen múltiples investigaciones (Llapa-Rodríguez, Trevizan, Shinyashiki y Mendes, 2009; Saldaña y Cornejo, 2017; Huaynate, 2019) que indican que los objetivos son alcanzados de forma eficaz cuando los trabajadores de una institución, en este caso los docentes, desarrollan un alto nivel de identificación y compromiso. En ese sentido, dicho compromiso del trabajador hacia la institución donde labora es el que se ha convertido en la actualidad uno de los fenómenos más estudiados en el campo de la administración y la gestión de recursos humanos. No obstante, en las instituciones educativas son pocas las políticas de revalorización, motivación, estímulos y mejora profesional hacia los docentes, lo que genera en ellos poco compromiso con el trabajo que realizan.

Bajo esa perspectiva, Porter, Steers, Mowday, y Boulian (1974) definen al compromiso organizacional como la fuerza relativa que conlleva a la identificación individual e implicación con una institución, la cual se caracteriza por el intenso deseo de mantenerse como integrante de una institución en particular, un acuerdo de mantener altos niveles de esfuerzo en pro de la institución y una creencia definitiva y aceptación de los valores y metas de la institución, que resulta de la orientación individual hacia la organización como un fin en sí mismo.

Del mismo modo, Steers (1977) definió al compromiso como aquella fuerza que le permite a un individuo involucrarse con la institución donde labora.

Al respecto, Meyer y Allen (1991) señalan que el compromiso organizacional es un estado psicológico; un proceso mediante el cual las metas de la organización y las metas individuales se integran. Ellos propusieron un concepto más completo del constructo compromiso organizacional y fueron quienes lo analizaron profundamente además de estudiar la identificación y la participación de los colaboradores con su organización. 
El compromiso organizacional se relaciona directamente con el sentido de pertenencia y la responsabilidad que posee cada persona con el lugar donde labora. Es trascendental tener trabajadores comprometidos y eficaces, en tanto que proporcionarían una alta eficacia y eficiencia organizacional.

Asimismo, un trabajador comprometido es una persona que está completamente involucrada y es entusiasta con la labor que realiza, está atraído e inspirado por su trabajo "Yo quiero hacer esto", entregado "Yo estoy dedicado al éxito de lo que estoy haciendo", y fascinado "Yo amo lo que estoy haciendo". Los empleados comprometidos se preocupan por el futuro de la organización y están dispuestos a invertir esfuerzo más allá del deber para que dicha organización tenga éxito, más allá de todo esto, es importante valorar el capital humano y el valor del trabajo que realizan (Rodríguez, 2017).

En relación con el desempeño docente, Montenegro (2003) realiza una aproximación conceptual acerca del término desempeño docente, considerando que es el cumplimiento cabal de sus responsabilidades el cual se encuentra determinado por factores asociados al mismo docente, a los estudiantes y su contexto. Asimismo, "el desempeño se ejerce en diferentes campos o niveles: el contexto sociocultural, el entorno institucional, el ambiente de aula y sobre el propio docente, mediante una acción reflexiva" (p.18). En ese sentido, es necesario evaluarlo para mejorar la calidad educativa y para cualificar la profesión docente. Por ello, la evaluación presenta funciones y características bien determinadas que se tienen en cuenta en el momento de la aplicación.

Por su parte, Robalino (2007) indica que el desempeño docente es el proceso de movilización de sus capacidades profesionales, su disposición personal y su responsabilidad social para articular relaciones significativas entre los componentes que impacta la formación de los alumnos, participar en gestión educativa, fortalecer una cultura institucional democrática e intervenir en el diseño, implementación y evaluación de políticas educativas locales y nacionales, para promover en los estudiantes aprendizajes y desarrollo de competencias y habilidades para la vida.

Otra definición importante la realiza el Ministerio de Educación (2012) al definir el desempeño como las acciones observables que realiza una persona, estas se pueden describir, así como evaluar y que dan cuenta de su competencia. Proviene del inglés performance o perform, y está relacionada con el logro de los aprendizajes previstos y el cumplimiento de las responsabilidades determinadas. Se entiende que la forma de realizar las mencionadas responsabilidades revela la competencia de base de la persona. En la definición de desempeño 
identificamos tres condiciones: 1) actuación observable, 2) una responsabilidad y 3) el logro de determinados resultados.

Bajo esa perspectiva, como antecedentes se encuentran Campana (2018) con la investigación "Compromiso organizacional y desempeño docente en la Institución Educativa Uriel García del Cusco, 2018” El objetivo de la investigación fue analizar la relación entre compromiso organizacional y desempeño docente. El enfoque fue cuantitativo, con un diseño no experimental; para la recolección de datos se aplicó el cuestionario de compromiso organizacional y desempeño docente en aula. Los resultados permitieron concluir con un nivel de confianza del 95\%, que, si existe relación directa y significativa entre compromiso organizacional y desempeño docente.

Del mismo modo está Ortiz (2018) con el estudio "Compromiso organizacional y desempeño en el aula del profesorado de instituciones educativas públicas”. El objetivo fue establecer la relación existente entre el compromiso organizacional y el desempeño docente desde la percepción de los profesores. La investigación tuvo un enfoque cuantitativo, de tipo descriptivo correlacional y un diseño no experimental. Los instrumentos utilizados para la recolección de datos fueron la Escala de Compromiso Organizacional y la Escala de Evaluación del Desempeño Docente. Los resultados indican una correlación directa de moderada intensidad entre el compromiso organizacional y el desempeño docente, siendo estadísticamente significativas a un nivel de probabilidad de 0,001 .

Asimismo, está Alvarado (2018) con la investigación "Compromiso organizacional y desempeño docente en las instituciones educativas del nivel primario de la red 02 -Puente Piedra, 2018". El objetivo fue determinar la relación entre el compromiso organizacional y el desempeño docente. La investigación se desarrolló bajo el enfoque cuantitativo; tuvo un diseño no experimental con un nivel descriptivo correlacional. Las conclusiones explican la existencia una correlación positiva y significativa entre las variables, con un coeficiente de 0.715 lo cual indica una correlación positiva considerable con un $\mathrm{p}=0.000<0.05$.

A partir del abordaje teórico y los antecedentes presentados se estableció como problema de investigación ¿Qué relación existe entre el compromiso organizacional y el desempeño docente en las Instituciones de Educación Básica del distrito de Las Piedras - 2018?

Este estudio se justifica desde el punto de vista institucional puesto que los resultados servirán al equipo directivo de las instituciones educativas focalizadas en la presente investigación para que apliquen acciones de reconocimiento, incentivo y apoyo para que puedan incrementar el compromiso de los docentes hacia sus instituciones y de esta manera puedan concretizar de mejor manera los objetivos que se planteen. Del mismo modo, desde el aspecto 
académico ayudará a conocer el nivel de desempeño que tienen los docentes, los directivos podrán ejecutar estrategias pedagógicas tales como el acompañamiento, trabajo colegiado y fortalecimiento de sus capacidades para que mejoren su práctica pedagógica y de esa forma los niveles de logro de aprendizaje de los estudiantes sean los más óptimos.

Por lo expuesto, se planteó como objetivo general de la presente investigación establecer la relación que existe entre el compromiso organizacional y el desempeño docente en las Instituciones de Educación Básica del distrito de Las Piedras - 2018.

\section{MÉTODO Y MATERIALES}

\section{Tipo y diseño de investigación}

El tipo de investigación fue no experimental (Hernández et al., 2010), ya que no se manipularon las variables de estudio, sino se observaron los fenómenos tal como se dan en su contexto natural para posteriormente analizarlos. El diseño metodológico que se utilizó en el presente estudio fue el descriptivo correlacional y transversal ya que nos centramos en medir y describir la relación entre las variables. La figura 1 representa el esquema del diseño de la presente investigación:

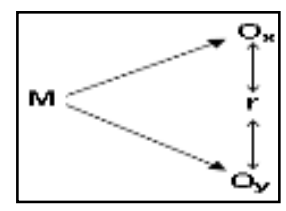

Figura 1. Esquema del diseño de investigación.

Dónde:

$\mathrm{M}=$ Muestra.

$\mathrm{O}_{\mathrm{x}}=$ Variable 1: Compromiso organizacional

$\mathrm{O}_{\mathrm{Y}}=$ Variable 2: Desempeño docente

$\mathrm{r}=$ Relación entre las variables de estudio.

\section{Población y muestra}

La población de estudio fue conformada por los todos los docentes que laboran en las 6 instituciones de educación básica pertenecientes al distrito de Las Piedras: Miguel Grau Seminario, Sudadero, Jorge Chávez Rengifo, Raúl Vargas Quiroz y Héroes de Illampu, los cuales son en total 106. En cuanto a la muestra, fue obtenida mediante un muestreo probabilístico estratificado, la cual fue de 83 docentes. En la tabla 1 se detalla la distribución de la muestra.

Tabla 1 
Distribución de la muestra

\begin{tabular}{lc}
\hline \multicolumn{1}{c}{ Institución Educativa } & $\mathrm{N}^{\circ}$ de docentes \\
\hline Miguel Grau Seminario & 21 \\
Sudadero & 9 \\
Jorge Chávez Rengifo & 19 \\
Raúl Vargas Quiroz & 15 \\
Héroes de Illampu & 19 \\
Total & 83 \\
\hline
\end{tabular}

Fuente: Elaboración propia

\section{Técnicas e instrumentos}

Para recoger información sobre el compromiso organizacional y desempeño docente se utilizó la técnica de la encuesta. En relación con los instrumentos, para medir la variable compromiso organizacional se utilizó el Cuestionario de Compromiso organizacional de Meyer y Allen, adaptado en el Perú por Oscar Martín Rivera Carrascal (2010), el cual evalúa el nivel de compromiso que los docentes presentan hacia su institución. Consta de tres dimensiones:

Compromiso afectivo: Es definido por Meyer y Allen (1991) como el lazo emocional que los trabajadores desarrollan y manifiestan hacia la institución, debido a que sus necesidades psicológicas y expectativas son satisfechas. Está relacionado con la percepción personal de las características objetivas y subjetivas de la organización, siendo fundamental para su satisfacción, motivación profesional y éxito organizacional (Neves, Graveto, Rodrigues, Marôco y Parreira, 2018).

El compromiso de continuidad: Se refiere a la conciencia del individuo sobre la inversión del tiempo y del esfuerzo que perderían si decidieran dejar de lado su institución para buscar otro empleo (Loli, 2006). Según afirman Llapa-Rodríguez, Trevizan, Shinyashiki y Mendes (2009) es caracterizado por aquel trabajador que se encuentra físicamente en su trabajo, pero que no invierte integralmente su capital humano, es decir, permanece no por la existencia de un vínculo emocional, sino porque los costos de no permanecer en él, pueden ser muy altos.

Compromiso normativo: Se refiere al deber ser, a la lealtad a la institución y la reciprocidad que tienen hacia ella (Arias, 2001, citado por Osorio, Ramos y Walteros, 2016). También es definido como la experimentación por parte del trabajador de un fuerte sentimiento de obligación de permanecer en la organización para la cual labora, revela los sentimientos de obligación del trabajador (Ríos, Téllez y Ferrer, 2010). 
Las mencionadas dimensiones se distribuyen en 21 ítems. La valoración de los ítems del cuestionario es mediante la escala de Likert (muy de acuerdo, de acuerdo, ni en acuerdo ni en desacuerdo, en desacuerdo o muy en desacuerdo).

Para medir la variable desempeño docente se aplicó el Cuestionario de Desempeño Docente, el cual fue adaptado del Marco del Buen Desempeño Docente elaborado por el Ministerio de Educación (2012). Está compuesto por 40 ítems a través de una escala de Likert (totalmente de acuerdo, de acuerdo, ni en acuerdo ni en desacuerdo, en desacuerdo y totalmente en desacuerdo). Se estructura en cuatro dimensiones:

Preparación para el aprendizaje de los estudiantes: Es entendida como el conjunto de actividades de planificación que realiza el docente vinculadas al trabajo pedagógico tales como programación curricular anual, unidades y sesiones de aprendizaje bajo un enfoque inclusivo e intercultural (Ministerio de Educación, 2012).

La enseñanza para el aprendizaje de los estudiantes: Se refiere al proceso de conducción de la enseñanza realizada por el docente y comprende el fomento de un clima de aula favorable, manejo de motivación y contenidos, conocimiento de diversas estrategias metodológicas, correcto proceso de evaluación y el uso adecuado de recursos didácticos (Ministerio de Educación, 2012).

La participación en la gestión de la escuela articulada a la comunidad: El docente tiene una participación activa en la institución, en las comisiones de trabajo como es: comisión de evaluación, comisión de elaboración y revisión del Proyecto Educativo Institucional y del Proyecto Curricular Institucional, etc. Realiza aportes significativos al diseño y desarrollo de proyectos pedagógicos y de orden institucional (Vargas, 2017).

El desarrollo de la profesionalidad y de la identidad docente: Es concebido como el proceso de reflexión sobre la práctica pedagógica que realiza el docente y la formación continua que realiza tanto de manera personal como colegiada.

\section{RESULTADOS}

Después de haber realizado la recolección de datos se procedió a sistematizarlos a través de tablas de frecuencia, para su análisis descriptivo, y matrices de correlación, para la prueba de hipótesis, las cuales se presentan a continuación.

Tabla 2 
Resultados descriptivos de la variable compromiso organizacional

\begin{tabular}{lcccc}
\hline \multicolumn{1}{c}{ Niveles } & Frecuencia & Porcentaje & Porcentaje válido & Porcentaje acumulado \\
\hline Muy bajo & 1 & 1,2 & 1,2 & 1,2 \\
Bajo & 13 & 15,7 & 15,7 & 16,9 \\
Medio & 21 & 25,3 & 25,3 & 42,2 \\
Alto & 40 & 48,2 & 48,2 & 90,4 \\
Muy alto & 8 & 9,6 & 9,6 & 100,0 \\
Total & 83 & 100,0 & 100,0 & \\
\hline
\end{tabular}

Fuente: Base de datos

De acuerdo con la tabla 2, el 48,2\% de los docentes presenta un compromiso organizacional alto, lo cual significa, según García, Useche y Schlesinger (2013) que se identifican con la institución donde laboran y sienten orgullo por ella, buscan que sus objetivos personales sean coherentes con los objetivos institucionales, toman entre dos o más alternativas la opción que más beneficie a la institución educativa, participa en el logro de planes y están involucrados en participar en las actividades programadas. Del mismo modo, el 25,3\% presenta un nivel de compromiso medio, también el $15,7 \%$ presenta un nivel bajo, por otro lado, el 9,6\% presenta un nivel de compromiso muy alto y solo el 1,2\% presenta un muy bajo compromiso organizacional, caracterizado por que existe indiferencia al logro de metas institucionales, escasa disposición a realizar esfuerzos en pro de ella y pocos deseos de mantenerse como integrante de la misma (Chiang, Gómez y Wackerling, 2016).

Tabla 3

Resultados descriptivos de la variable desempeño docente

\begin{tabular}{lcccc}
\hline \multicolumn{1}{c}{ Niveles } & Frecuencia & Porcentaje & Porcentaje válido & Porcentaje acumulado \\
\hline Deficiente & 1 & 1,2 & 1,2 & 1,2 \\
En proceso & 22 & 26,5 & 26,5 & 27,7 \\
Satisfactorio & 50 & 60,2 & 60,2 & 88,0 \\
Destacado & 10 & 12,0 & 12,0 & 100,0 \\
Total & 83 & 100,0 & 100,0 & \\
\hline
\end{tabular}

Fuente: Base de datos

Según la tabla 3, la mayor parte de los docentes $(60,2 \%)$ presenta un nivel de desempeño satisfactorio, lo que se caracteriza porque los docentes planifican las estrategias, recursos y materiales, realizan el proceso de enseñanza teniendo en cuenta las particularidades, ritmos y estilos de aprendizaje, además de ejecutar los procesos pedagógicos y didácticos, participan activamente en la gestión de la institución educativa y reflexiona sobre su práctica pedagógica para mejorar su desempeño (Andia, 2018). Por otro lado, el 26,5\% se encuentra en proceso de llegar a un buen desempeño, el $12 \%$ presenta un desempeño destacado y finalmente el 1,2\% presenta un nivel de desempeño deficiente, caracterizado según Oscco (2015) por la deficiente 
preparación y ejecución de las sesiones de aprendizaje, desconocimiento de las características de los estudiantes, mínima participación en actividades institucionales y pocos deseos de superación personal.

Tabla 4

Matriz de correlación entre las variables compromiso organizacional y desempeño docente

\begin{tabular}{|c|c|c|c|c|}
\hline & & & $\begin{array}{c}\text { Compromiso } \\
\text { organizacional }\end{array}$ & $\begin{array}{c}\text { Desempeño } \\
\text { docente }\end{array}$ \\
\hline \multirow{5}{*}{$\begin{array}{l}\text { Rho de } \\
\text { Spearman }\end{array}$} & \multirow{2}{*}{$\begin{array}{l}\text { Compromiso } \\
\text { organizacional }\end{array}$} & $\begin{array}{l}\text { Coeficiente de } \\
\text { correlación }\end{array}$ & 1,000 &, $724^{* *}$ \\
\hline & & Sig. (bilateral) & 253 & $\begin{array}{l}, 000 \\
83\end{array}$ \\
\hline & \multirow{3}{*}{$\begin{array}{l}\text { Desempeño } \\
\text { docente }\end{array}$} & $\begin{array}{l}\text { Coeficiente de } \\
\text { correlación }\end{array}$ &, $724^{* *}$ & 1,000 \\
\hline & & Sig. (bilateral) &, 000 & $\cdot$ \\
\hline & & $\mathrm{N}$ & 83 & 253 \\
\hline
\end{tabular}

**. La correlación es significativa en el nivel 0,01 (2 colas).

Fuente: Base de datos

La tabla 4 nos muestra que el coeficiente de correlación rho de Spearman entre las variables compromiso organizacional y desempeño docente es de 0,724 con un p-valor inferior al nivel de significancia $(\mathrm{p}=0,000<0,05)$. Por lo expuesto, se concluye que existe una correlación positiva fuerte entre las variables analizadas. Esto quiere decir que mientras mayor sea el compromiso organizacional de los docentes, mayor será su desempeño y viceversa.

Tabla 5

Matriz de correlación entre las dimensiones compromiso afectivo, de continuidad y normativo y la variable desempeño docente

\begin{tabular}{cclc}
\hline & & & Desempeño docente \\
\hline \multirow{4}{*}{ Rho de } & Compromiso & Coeficiente de correlación &, $569 * *$ \\
Spearman & afectivo & Sig. (bilateral) &, 000 \\
& \multirow{2}{*}{ Compromiso de } & Coeficiente de correlación & 83 \\
\cline { 2 - 4 } & continuidad & Sig. (bilateral) &, $669^{* *}$ \\
& Compromiso & Coeficiente de correlación &, 000 \\
& normativo & Sig. (bilateral) &, 63 \\
& & &, $004 *$
\end{tabular}


Fuente: Base de datos

**. La correlación es significativa en el nivel 0,01 ( 2 colas).

En la tabla 5 se puede apreciar que existe correlación positiva considerable entre las dimensiones compromiso afectivo ( $\mathrm{rs}=0,569 ; \mathrm{p}=0,000<0,05)$, compromiso de continuidad ( $r s=0,669 ; \mathrm{p}=0,000<0,05)$ y compromiso normativo ( $\mathrm{rs}=0,664 ; \mathrm{p}=0,000<0,05$ ) y la variable desempeño docente. En ese sentido, se puede ver que la dimensión de la variable compromiso organizacional que se relaciona más con el desempeño docente es el compromiso de continuidad.

\section{DISCUSIÓN}

Al observar los resultados generales obtenidos, lo primero que hallamos es que los docentes de las Instituciones de Educación Básica se caracterizan por presentar altos niveles de compromiso organizacional. Estos resultados indican que los docentes se identifican con la institución donde laboran sus objetivos trazados, involucrándose en las actividades, aunque si hubiera otras opciones de trabajo, analizarían su permanencia. Los resultados hallados difieren de los resultados obtenidos por Rivera (2010), Campana (2018), Alvarado (2018) y Ortiz (2018) quienes hallaron en sus investigaciones que el compromiso organizacional se encuentra en un término medio.

En relación con el desempeño docente, la mayor parte de los docentes de las Instituciones de Educación Básica se caracteriza por presentar un nivel de desempeño satisfactorio. Estos resultados indican que la preparación para el aprendizaje, la enseñanza, la participación en la gestión de la escuela y el desarrollo de la profesionalidad y de la identidad docente es adecuada, sin embargo, existe un considerable porcentaje de docentes que se encuentra en inicio y proceso de lograr un desempeño pertinente por lo que es necesario realizar el acompañamiento pedagógico para fortalecer sus capacidades. Los resultados presentados coinciden con la investigación de Macavilca (2018) quien halló que la mayoría de docentes presentaban un desempeño adecuado.

$\mathrm{Al}$ analizar la correlación entre las variables compromiso organizacional y desempeño docente, se puede apreciar que el coeficiente de correlación rho de Spearman entre las variables compromiso organizacional y desempeño docente es de 0,724 con un p-valor inferior al nivel de significancia $(\mathrm{p}=0,000<0,05)$. Esto quiere decir que mientras mayor sea el compromiso organizacional de los docentes, mayor será su desempeño y viceversa. En ese sentido, el estar comprometidos con la labor que realizamos y la institución en la cual laboramos conlleva que 
podamos desempeñarnos de la mejor manera puesto que nos pondremos la camiseta institucional y contribuiremos desde nuestro lado con concretizar los objetivos y metas que las instituciones se hayan planteado. No obstante, si los docentes no se encuentran comprometidos e identificados con la institución, no lograrán desempeñarse adecuadamente lo que conllevará a la pérdida de la calidad educativa. Estos resultados coinciden con la investigación de Ramos (2005) quien halló que existe una correlación positiva entre el compromiso organizacional y el desempeño docente y existe correlación entre las dimensiones compromiso afectivo, compromiso normativo y de continuidad con la variable desempeño docente. Del mismo modo es corroborado por la investigación Gómez (2015) que indica que existe evidencia empírica para afirmar que existe una correlación directa y significativa entre el compromiso organizacional y el desempeño docente. De la misma forma, coincide con la investigación de Leyva (2016) quien halló que existe relación positiva entre la variable de compromiso organizacional y el desempeño docente. Asimismo, guarda relación con el trabajo de Guarniz (2014) quien estableció a partir de su estudio que existe correlación significativa entre las variables compromiso y desempeño docente. Del mismo modo coincide con el estudio de Campana (2018) quien halló, con un nivel de confianza del 95\%, que sí existe relación directa y significativa entre compromiso organizacional y desempeño docente en aula en la Institución Educativa Uriel García del Cusco, 2018, según la prueba de Chi Cuadrado. Por otra parte, es corroborado por la investigación de Ortiz (2018) quien determinó que existe una relación directa de moderada intensidad y estadísticamente significativa $(r=0,53)$ entre el compromiso organizacional y el desempeño docente del profesorado de las Instituciones Educativas Públicas —Nicolás de Piérola, —Luis Montfort, —José A. Quiñones y —Leoncio Prado de Ate-Vitarte durante el año 2016. Finalmente coincide con la investigación de Alvarado (2018) quien determinó la existencia de una correlación positiva y significativa entre las variables, con un coeficiente de 0.715 lo cual indica una correlación positiva considerable y un $\mathrm{p}=0.000<0.05$.

Por último, se pudo hallar que existe correlación positiva considerable entre las dimensiones compromiso afectivo ( $\mathrm{rs}=0,569 ; \mathrm{p}=0,000<0,05)$, compromiso de continuidad ( $r s=0,669 ; \mathrm{p}=0,000<0,05)$ y compromiso normativo ( $\mathrm{rs}=0,664 ; \mathrm{p}=0,000<0,05)$ y la variable desempeño docente. En ese sentido, se puede ver que la dimensión de la variable compromiso organizacional que se relaciona más con el desempeño docente es el compromiso de continuidad. Estos resultados coinciden con las investigaciones de Ortiz (2018), Macavilca (2018) y Baltazar (2017) los cuales hallaron que las dimensiones de la variable compromiso afectivo se correlacionan directa y significativamente con el desempeño docente. 


\section{CONCLUSIONES}

Se determinó que existe una correlación positiva fuerte entre el compromiso organizacional y el desempeño docente en las Instituciones de Educación Básica del distrito de Las Piedras, 2018, al encontrar estadísticamente un coeficiente de correlación rho de Spearman de 0,724 con un $\mathrm{p}$-valor inferior al nivel de significancia $(\mathrm{p}=0,000<0,05)$.

Asimismo, se estableció que existe una correlación positiva considerable entre la dimensión compromiso afectivo y el desempeño docente en las Instituciones de Educación Básica del distrito de Las Piedras, 2018, al encontrar un coeficiente de correlación rho de Spearman de 0,569 con un $p$-valor inferior al nivel de significancia $(p=0,000<0,05)$.

En la misma perspectiva, se encontró una correlación positiva considerable entre la dimensión compromiso de continuidad y el desempeño docente en las Instituciones de Educación Básica del distrito de Las Piedras, 2018, al encontrar un coeficiente de correlación rho de Spearman de 0,669 con un $p$-valor inferior al nivel de significancia $(p=0,000<0,05)$.

De igual manera, existe una correlación positiva considerable entre la dimensión compromiso normativo y el desempeño docente en las Instituciones de Educación Básica del distrito de Las Piedras, 2018, al encontrar un coeficiente de correlación rho de Spearman de 0,664 con un $p$-valor inferior al nivel de significancia $(\mathrm{p}=0,000<0,05)$.

Estos resultados suponen una contribución a los directores y subdirectores de Las diferentes instituciones educativas puesto que fomentará en ellos la capacidad de gestionar, motivar y comprometer a los docentes que tienen a cargo para que se desempeñen eficientemente cumpliendo con los dominios, competencias y desempeños establecidos en el Marco del Buen Desempeño Docente.

\section{REFERENCIAS BIBLIOGRÁFICAS}

Alvarado, M. (2018). Compromiso organizacional y desempeño docente en las instituciones educativas del nivel primario de la red 02 -Puente Piedra, 2018. (Tesis de Maestría). Universidad César Vallejo, Lima, Perú.

Andia, E. (2018). Desempeño docente y rendimiento académico en los estudiantes del Primer Grado de Educación Secundaria de la Institución Educativa Pública "Esmeralda de los Andes”. Huanta, 2017. (Tesis de Maestría). Universidad César Vallejo, Ayacucho, Perú.

Arias, F. (2001). El compromiso personal hacia la organización y la intención de permanencia: algunos factores para su incremento. Contaduría y Administración, 200. Recuperado de http://www.ejournal.unam.mx/rca/200/RCA20001.pdf 
Baltazar, N. (2017). Compromiso organizacional y desempeño docente en colegios públicos de la cuenca Nor Alta; UGEL N 15, Huarochirí, 2017. (Tesis de Maestría en Educación). Universidad César Vallejo, Lima, Perú.

Campana, M. (2018). Compromiso organizacional y desempeño docente en la Institución Educativa Uriel García del Cusco, 2018. (Tesis de Maestría en Educación). Universidad Nacional de San Antonio Abad del Cusco, Cusco, Perú.

Chiang, M., Gómez, N. y Wackerling, L. (2016). Organization commitment of rural municipal workers. Ciencia \& trabajo, 18(56), 134-138. Recuperado de https://scielo.conicyt.cl/pdf/cyt/v18n56/art10.pdf

García, L., Useche, M. y Schlesinger, M. (2013). Empowerment and organizational commitment of teachers in the University researcher Zulia. REDHECS, 16(9), 6-19. Recuperado de http://ojs.urbe.edu/index.php/redhecs/article/view/500/427

Gómez, A. (2015). Gestión del compromiso institucional y el desempeño docente del profesorado de la Universidad Peruana los Andes. (Tesis de Maestría). Universidad Nacional de Educación Enrique Guzmán y Valle, Lima, Perú.

Guarniz, A. (2014). Satisfacción laboral, compromiso institucional y desempeño docente de los profesores de la Facultad de Medicina de la Universidad Privada Antenor Orrego, 2012. (Tesis de Maestría). Universidad Privada Antenor Orrego, Trujillo, Perú.

Hernández, R., Fernández, C. y Baptista, P. (2010). Metodología de la investigación. México: Mc Graw Hill.

Huaynate, E. (2019). Relación del compromiso organizacional y desempeño docente en las instituciones educativas públicas de Educación Básica Regular del Distrito de Huánuco 2017. (Tesis Doctoral), Universidad Nacional Hermilio Valdizán, Huánuco, Perú.

Leyva, M. (2016). Compromiso organizacional y desempeño docente en la I.E. 2027 José María Arguedas UGEL 02 2015. (Tesis de Maestría). Universidad César Vallejo, Lima, Perú.

Llapa-Rodríguez, E., Trevizan, M., Shinyashiki, T. y Mendes, I. (2009). Comprometimento organizacional e profissional da equipe de saúde. Enfermería Global, 8(3), 1-16. Recuperado de https://revistas.um.es/eglobal/article/view/75231/72971

Loli, A. (2006). Compromiso organizacional de los trabajadores de una Universidad Pública de Lima y su relación con algunas variables demográficas. Revista IIPSI, 9(1), 37-67. Recuperado de https://revistasinvestigacion.unmsm.edu.pe/index.php/psico/article/view/4028/3208 
Macavilca, A. (2018). Compromiso organizacional y desempeño docente en las instituciones educativas del distrito de Huarochirí, 2018. (Tesis de Maestría en Educación). Universidad César Vallejo, Lima, Perú.

Meyer, J. y Allen, N. (1991). A three component conceptualization of organizational commitment. Human Resource Management Review. 1, 61-89.

Ministerio de Educación (2012). Marco del Buen Desempeño Docente. Lima: MINEDU.

Montenegro, I. (2003). Evaluación del desempeño docente. Fundamentos, modelos e instrumentos. Colombia: Edit. Coop. Editorial Magisterio.

Neves, T., Graveto, J., Rodrigues, V., Marôco, J. y Parreira, P. (2018). Organizational commitment, psychometric qualities and invariance of the Meyer and Allen Questionnaire for Portuguese Nurses. Revista Latino-Americana de Enfermagem, 26, 111. Recuperado de http://www.scielo.br/pdf/rlae/v26/es_0104-1169-rlae-26-e3021.pdf

Ortiz, M. (2018). Compromiso organizacional y desempeño en el aula del profesorado de instituciones educativas públicas. (Tesis de Maestría en Educación). Universidad Nacional de Educación “Enrique Guzmán y Valle”, Lima, Perú.

Oscco, R. (2015). Optimización del desempeño docente en la forma de atención semipresencial y el logro de competencias en los estudiantes del centro piloto madre Teresa de Calcuta de educación básica alternativa de San Juan de Lurigancho, 2014. (Tesis de Doctorado). Universidad Nacional de Educación Enrique Guzmán y Valle, Lima, Perú.

Osorio, L., Ramos, E. y Walteros, D. (2016). Personal values and organizational commitment: more than a simple relationship. Revista de Psicología y Ciencias del Comportamiento. $7(2), 33-51$.

Porter, L., Steers, R., Mowday, R., y Boulian, R. (1974). The Measurement of Organizational Commitment". Journal of Vocational Behavior, 14, 224-247.

Ramos, A. (2005). El compromiso organizacional y su relación con el desempeño docente de los profesores del programa universitario de inglés de la Universidad de Colima. (Tesis de Maestría). Universidad de Colima, Villa de Álvarez, Colima.

Ramírez, A. y Domínguez, L. (2012). The organizational climate and organizational commitment heis in Puerto Vallarta. Investigación Administrativa, 109(41), 21-30. Recuperado de https://www.redalyc.org/pdf/4560/456045214002.pdf

Ríos, M., Téllez, M. y Ferrer, J. (2010. Empowerment as a predictive indicator of organizational commitment in SMEs. Contaduría y administración, 231, 103-125. Recuperado de http://www.scielo.org.mx/pdf/cya/n231/n231a6.pdf 
Rivera, O. (2010). Compromiso organizacional de los docentes de una institución educativa privada de Lima Metropolitana y su correlación con variables demográficas. (Tesis de Maestría). Pontificia Universidad Católica del Perú, Lima, Perú.

Robalino, M. (2007). Los docentes pueden hacer la diferencia: apuntes acerca del desarrollo profesional y el protagonismo docente. Seminario internacional llevado a cabo en Chile.

Rodríguez, J. (2016). Compromiso organizacional docente de una Institución Educativa Pública Técnico Productiva del AA.HH. Nueva Magdalena del distrito Cillería de Coronel Portillo región Ucayali. (Tesis de Licenciatura). Universidad Marcelino Champagnat, Lima, Perú.

Saldaña, C. y Cornejo, F. (2017). Relación entre el compromiso organizacional y el desempeño laboral del personal empleado de la Universidad Peruana Unión, Lima, 2016. (Tesis de Licenciatura). Universidad Peruana Unión, Lima, Perú.

Steers, R. (1977). Antecedents and outcomes of organizational commitment. Administrative Science Quarterly. 22, 46-56.

Vargas, W. (2017). La supervisión educativa y su relación con el Marco del buen desempeño docente en área de Educación para el Trabajo, en Instituciones Educativas del Distrito de Lurigancho, 2016. (Tesis de Licenciatura). Universidad Nacional de Educación Enrique Guzmán y Valle, Lima, Perú.

\section{Conflicto de intereses / Competing interests:}

Los autores declaran que no incurre en conflictos de intereses.

Rol de los autores / Authors Roles:

Edwin Estrada. conceptualización, curación de datos, análisis formal, adquisición de fondos, investigación, metodología, administración del proyecto, recursos, software, supervisión, validación, visualización, escritura - శึ preparación del borrador original, escritura - revisar \& amp; edición.

Helen Mamani: conceptualización, investigación, metodología, administración del proyecto, recursos, software, supervisión, validación, visualización, escritura - preparación del borrador original, escritura - revisar \& amp; edición.

Fuentes de financiamiento / Funding:

Los autores declaran que no recibió un fondo específico para esta investigación.

Aspectos éticos / legales; Ethics / legals:

Los autores declaran no haber incurrido en aspectos antiéticos, ni haber omitido aspectos legales en la realización de la investigación. 